https://doi.org/10.15407/ujpe63.7.616

V.I. ROMANENKO, O.G. UDOVYTSKA, V.M. KHODAKOVSKY, L.P. YATSENKO

Institute of Physics, Nat. Acad. of Sci. of Ukraine

(46, Nauky Ave., Kyiv 03028,Ukraine; e-mail: vr@iop.kiev.ua)

\title{
ATOMIC MOMENTUM DIFFUSION IN THE FIELD OF COUNTER-PROPAGATING STOCHASTIC LIGHT WAVES
}

\begin{abstract}
The momentum diffusion of atoms in the field of two counter-propagating stochastic light waves, one of which reproduces the other one with a certain time delay, has been studied. It is shown that the parameters of atom-field interaction, at which the light pressure force is maximum, correspond to the increasing momentum diffusion coefficient. In the case of highintensity field described by the stochastic field model, the momentum diffusion coefficient was found to be proportional to the square root of the field autocorrelation time. The wave function describing the inner state of atoms is modeled, by using the Monte-Carlo method. Numerical calculations are carried out for cesium atoms.
\end{abstract}

Keywords: light pressure, stochastic fields, Monte-Carlo method, wave function.

\section{Introduction}

Till now, the force of light pressure on atoms in the field of counter-propagating correlated stochastic waves has been studied in a number of works [16]. Like the "bichromatic force", i.e. the force of light pressure on atoms in the field of counter-propagating bichromatic waves [7-9], the force of light pressure on an atom in the field of counter-propagating light waves can significantly exceed the maximum of the light pressure force on an atom in the field of a running monochromatic wave

$F_{s p}=\hbar k \gamma / 2$,

where $k=2 \pi / \lambda$ is the wave vector and $\lambda$ the wavelength of electromagnetic radiation, and $\gamma$ is the inverse lifetime of an atom in the excited state (the two-level atomic model is assumed).

It should be noted that the momentum diffusion of atoms, which always accompanies the light pressure on atoms [10], has not been studied yet in the case of stochastic light waves. In this work, we have tried to partially fill this lacuna by analyzing the dependence of the statistical characteristics of an atomic ensemble on the parameters of the interaction between the atoms and the stochastic field. Our research is based on the computer simulation of the atomic motion

(c) V.I. ROMANENKO, O.G. UDOVYTSKA, V.M. KHODAKOVSKY, L.P. YATSENKO, 2018 in the field of stochastic waves (the stochastic field model), by using the Newton equations, which was followed by the calculation of the statistical characteristics of the atomic ensemble. The interaction between the atom and the field is modeled in the framework of the two-level atomic excitation scheme. The density matrix, which is required to calculate the force, is determined from the probability amplitudes of the state vector, by using the Monte-Carlo method for the wave function [11]. The numerical simulation is carried out for the parameters corresponding to the interaction of cesium atoms with the light field.

The structure of the work is as follows. In Section 2, a model of the interaction between the atom and the field is discussed. In Section 3, the equations describing the atom-field interaction and the evolution of the state vector are formulated, and the method used for calculating the statistical characteristics of the atomic ensemble is described. The results obtained are quoted and their discussion is made in Section 4 . A brief summary of the research results is made in Conclusions.

\section{Model of the Interaction between an Atom and the Field}

Let an atom move along the $z$-axis in the field of two counter-propagating waves

$\mathbf{E}=\frac{1}{2} \mathbf{e}\left[E_{0}(t-z / c) \exp (i \omega t-i k z)+\right.$ ISSN 2071-0194. Ukr. J. Phys. 2018. Vol. 63, No. 7 
$\left.+E_{0}(t+z / c) \exp (i \omega t+i k z)\right]+$ K.c.

where $\mathbf{e}$ is the polarization vector. At the coordinate $z$, where the atom is located, one of the waves reproduces the other one with a certain time delay $\tau=2 z / c$. The coordinate origin is selected so that the field strengths of counter-propagating waves coincide at the point $z=0$.

Laser radiation is described in the framework of the chaotic field model $[12,13]$. Namely, the real and imaginary parts of the complex-valued field amplitude fluctuate independently, their average values equal zero, an the corresponding autocorrelation functions look like

$\begin{aligned}\left\langle\Re E_{0}(t) \Re E_{0}\left(t^{\prime}\right)\right\rangle & =\frac{1}{2}\left\langle\left|E_{0}\right|^{2}\right\rangle e^{-G\left|t-t^{\prime}\right|}, \\ \left\langle\Im E_{0}(t) \Im E_{0}\left(t^{\prime}\right)\right\rangle & =\frac{1}{2}\left\langle\left|E_{0}\right|^{2}\right\rangle e^{-G\left|t-t^{\prime}\right|},\end{aligned}$

where the angle brackets $\langle\ldots\rangle$ denote the averaging over the ensemble of states obtained in the course of a stochastic process, $G$ is the inverse autocorrelation time of the field amplitude, and $\left\langle\left|E_{0}\right|^{2}\right\rangle=$ $\left\langle E_{0}(t) E_{0}^{*}(t)\right\rangle$ is time-independent. Figure 1 illustrates the interaction of an atom with this field. A stochastic process with the exponential time dependence of the correlation function of type $(3),(4)$ is called the "colored noise" [14].

The interaction of the atom with the field is characterized by the Rabi frequency $\Omega_{0}=-\mathbf{d e} \sqrt{\left\langle\left|E_{0}\right|^{2}\right\rangle} / \hbar$, where $\mathbf{d}$ is the matrix element of the atomic dipole moment operator corresponding to the working transition.

The corresponding illustrative calculations are carried out for an atom ${ }^{133} \mathrm{Cs}$. The wavelength of the working transition $6^{2} S_{1 / 2}-6^{2} P_{3 / 2}$ equals $\lambda=$ $=852.35 \mathrm{~nm}$, the spontaneous emission rate $\gamma=$ $=2 \pi \times 5.18 \mathrm{MHz}$, and the Doppler cooling limit of atoms $T_{\mathrm{D}}=124.39 \mu \mathrm{K}[15]$.

\section{Basic Equations}

An atom in field (2) is subjected to the action of light pressure with the force $[10,15]$

$F=\left(\varrho_{12} \mathbf{d}_{21}+\varrho_{21} \mathbf{d}_{12}\right) \frac{\partial \mathbf{E}}{\partial z}$,

where $\mathbf{d}_{12}$ and $\mathbf{d}_{21}$ are the matrix elements of the dipole moment operator, and $\varrho_{12}$ and $\varrho_{21}$ are the elements of the density matrix $\varrho$. Under the action of

ISSN 2071-0194. Ukr. J. Phys. 2018. Vol. 63, No. 7

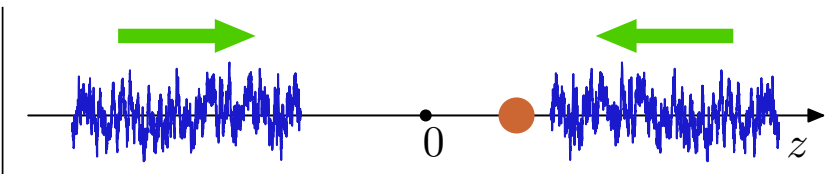

Fig. 1. Scheme of the interaction between an atom (exhibited as a circle) and the field of counter-propagating waves. One of the waves reproduces the other one. The stochastic amplitude envelopes are shown

force (5), the atom moves according to the second Newton law

$\dot{v}=F / m$

where $m$ is the mass of the atom, and $v=\dot{z}$ is its velocity. To find the changes of the atomic velocity and coordinate in time from Eqs. (5) and (6), the matrix of the atomic density has to be known. Its elements for the atomic state vector

$|\psi\rangle=c_{1}|1\rangle+c_{2} e^{-i \omega_{0} t}|2\rangle$

are calculated by the formulas

$\varrho_{12}=c_{1} c_{2}^{*} e^{i \omega_{0} t}, \quad \varrho_{21}=c_{2} c_{1}^{*} e^{-i \omega_{0} t}$,

where the probability amplitudes $c_{1}$ and $c_{2}$ of the atomic states $|1\rangle$ and $|2\rangle$, respectively, are determined from the Schrödinger equation

$i \hbar \frac{d}{d t}|\psi\rangle=H|\psi\rangle$.

The state vector is calculated, by making allowance for quantum jumps that are responsible for the atomic spontaneous emission (the Monte-Carlo method for the wave function [11]). The Hamiltonian in the Schrödinger equation (9) looks like

$H=H_{0}+H_{\text {int }}+H_{\text {rel }}$.

Here, the term

$H_{0}=\hbar \omega_{0}|2\rangle\langle 2|$

describes the atom in the absence of the field and relaxation; the term

$H_{\text {int }}=-\mathbf{d}_{12}|1\rangle\left\langle 2\left|\mathbf{E}(t)-\mathbf{d}_{21}\right| 2\right\rangle\langle 1| \mathbf{E}(t)$,

where $\mathbf{d}_{12}$ is the matrix element of the electric dipole moment operator for the transition between states $|1\rangle$ 
and $|2\rangle$, describes the interaction between the atom and the field; and the term

$H_{\mathrm{rel}}=-\frac{i \hbar \gamma}{2}|2\rangle\langle 2|$

describes the relaxation due to the spontaneous radiation emission.

Let the state vector be $|\psi(t)\rangle$ at the time moment $t$. The wave function $|\psi(t+\Delta t)\rangle$ at the time moment $t+\Delta t$ is determined in two stages [11].

1. From Eq. (9), it follows that, after a small time interval $\Delta t$, the state vector $|\psi(t)\rangle$ transforms into

$\left|\psi^{(1)}(t+\Delta t)\right\rangle=\left(1-\frac{i \Delta t}{\hbar} H\right)|\psi(t)\rangle$.

Since Hamiltonian (10) is non-Hermitian, the state vector $\left|\psi^{(1)}(t+\Delta t)\right\rangle$ is not normalized. At a small $\Delta t$, the square of its norm equals

$\left\langle\psi^{(1)}(t+\Delta t) \mid \psi^{(1)}(t+\Delta t)\right\rangle=1-\Delta P$,

where

$\Delta P=\frac{i \Delta t}{\hbar}\left\langle\psi(t)\left|H-H^{+}\right| \psi(t)\right\rangle=\gamma \Delta t\left|c_{2}\right|^{2}$.

2. At the second stage, let us take into account that a quantum jump is possible. If the random variable $\epsilon$, which is uniformly distributed between zero and one, is larger than $\Delta P$, there is no spontaneous emission, and we simply normalize the state vector:

$|\psi(t+\Delta t)\rangle=\frac{\left|\psi^{(1)}(t+\Delta t)\right\rangle}{\sqrt{1-\Delta P}}$ if $\Delta P<\epsilon$.

But if $\epsilon<\Delta P$, the atom spontaneously emits a photon and transits into the ground state:

$|\psi(t+\Delta t)\rangle=|1\rangle$ if $\Delta P>\epsilon$.

Equations for the probability amplitudes follow from the Schrödinger equation (9). In the rotatingwave approximation, i.e. neglecting the rapidly oscillating terms $\sim e^{ \pm 2 i \omega_{0} t}[16]$, they acquire the form

$\frac{d}{d t} c_{1}=-\frac{i}{2}\left(\Omega_{1} e^{-i k z}+\Omega_{2} e^{i k z}\right) c_{2} e^{-i \delta t}$,

$\frac{d}{d t} c_{2}=-\frac{i}{2}\left(\Omega_{1}^{*} e^{i k z}+\Omega_{2}^{*} e^{-i k z}\right) c_{1} e^{i \delta t}-\frac{\gamma}{2} c_{2}$,

where $\Omega_{1}=\Omega(t-z / c), \Omega_{2}=\Omega(t+z / c), \Omega(t)=$ $=-\mathbf{d}_{12} \mathbf{e} E_{0}(t) / \hbar$, and $\delta=\omega_{0}-\omega$.

618
As follows from Eqs. (3) and (4), the autocorrelation functions for the real and imaginary parts of $\Omega(t)$ equal

$\left\langle\Re \Omega(t) \Re \Omega\left(t^{\prime}\right)\right\rangle=\frac{1}{2} \Omega_{0}^{2} e^{-G\left|t-t^{\prime}\right|}$,
$\left\langle\Im \Omega(t) \Im \Omega\left(t^{\prime}\right)\right\rangle=\frac{1}{2} \Omega_{0}^{2} e^{-G\left|t-t^{\prime}\right|}$,

where $\Omega_{0}=\sqrt{\left\langle|\Omega|^{2}\right\rangle}$. Furthermore,

$\left\langle\Re \Omega(t) \Im \Omega\left(t^{\prime}\right)\right\rangle=0$,

$\langle\Re \Omega(t)\rangle=\langle\Im \Omega(t)\rangle=0$.

The found wave function makes it possible, by using Eqs. (5) and (8), to calculate the force of light pressure on the atom and describe the motion of this atom by simultaneously integrating the Schrödinger and Newton equations. After averaging the force expression (5) over the field oscillation period $2 \pi / \omega_{0}$, we have

$F=\frac{\hbar k \operatorname{Im}\left[c_{1} c_{2}^{*} e^{i \delta t}\left(\Omega_{1}^{*} e^{i k z}-\Omega_{2}^{*} e^{-i k z}\right)\right]}{\left|c_{1}\right|^{2}+\left|c_{2}\right|^{2}}$.

The chaotic field is modeled by the OrnsteinUhlenbeck process $\Xi(t)$ [14]. Its autocorrelation function

$\left\langle\Xi(t) \Xi\left(t^{\prime}\right)\right\rangle=B G e^{-G\left|t-t^{\prime}\right|}$

at $\Omega_{0}=\sqrt{2 B G}$ coincides with Eq. (21), if $\Xi(t)=$ $=\operatorname{Re} \Omega(t)$, and with Eq. (22), if $\Xi(t)=\operatorname{Im} \Omega(t)$. This process is a solution of the equation

$\frac{d}{d t} \Xi(t)=-G \Xi(t)+G \xi(t)$,

where $\xi(t)$ is the Gaussian white noise, which is characterized by the following mean values over the ensemble:

$\left\langle\xi(t) \xi\left(t^{\prime}\right)\right\rangle=2 B \delta\left(t-t^{\prime}\right), \quad\langle\xi(t)\rangle=0$.

Here, $\delta(t)$ is the Dirac delta-function. The OrnsteinUhlenbeck process is a "colored noise" [14], which transforms into the white one at $G \rightarrow \infty$.

We simulated the Ornstein-Uhlenbeck process by the sequence $[17,18]$

$\Xi\left(t_{j+1}\right)=\Xi\left(t_{j}\right) \exp (-G \Delta t)+h\left(t_{j}\right)$.

Here, $\Delta t=t_{j+1}-t_{j}$, and $h\left(t_{j}\right)$ are distributed according to the Gaussian law with the first moment equal to zero, and the second moment equal to

$\left\langle h\left(t_{j}\right)^{2}\right\rangle=D G\left(1-e^{-2 G \Delta t}\right)$.

ISSN 2071-0194. Ukr. J. Phys. 2018. Vol. 63, No. 7 


\section{Results and Discussion}

Cesium atoms ${ }^{133} \mathrm{Cs}$ were chosen for the numerical simulation, because they allow a two-level system with the atom-field interaction to be realized [15]. Furthermore, their atomic mass is large enough for their average velocity not to change much within the time interval required for the atomic velocity distribution typical of a diffusion process to be established. The force of light pressure and the coefficient of momentum diffusion at the point with the coordinate $z$ depend on the time delay

$\tau=\frac{2 z}{c}$

between the counter-propagating stochastic waves at this point.

We calculated the diffusion coefficient of atomic velocities, $D_{v}$, which is more illustrative than the momentum diffusion coefficient $D$. In view of the relation $p=m v$ between the momentum $p$ and the velocity $v$ of the atom, it is easy to express one of the constants in terms of the other one: $D=m^{2} D_{v}$. The time, $t$, dependence of the mean square deviation $\Delta v$ of the atomic velocity from its mean value is given by the formula

$\Delta v=\sqrt{2 D_{v} t}$.

The values of the light pressure force and the velocity diffusion coefficient were calculated from the time dependences of the mean velocity and the mean square deviation of atomic velocities from the mean value for an ensemble of cesium atoms. Some examples of calculation results obtained for $\tau=2.3 \mathrm{~ns}(z=0.35 \mathrm{~m})$ are shown in Fig. 2.

In the case of low-intensity counter-propagating waves, $\Omega_{0} \ll \gamma$, and one-dimensional model, which is considered here, the momentum diffusion coefficient was calculated analytically in work [6]:

$D=(\hbar k)^{2} \Omega_{0}^{2} \frac{G+\frac{\gamma}{2}}{\left(G+\frac{\gamma}{2}\right)^{2}+\delta^{2}}$.

This result is used to test the software program of atomic motion simulation in the field of counterpropagating stochastic waves.

The dependences exhibited in Fig. 2 make it possible to determine the average acceleration of the atom (and, hence, the average force acting on the latter)
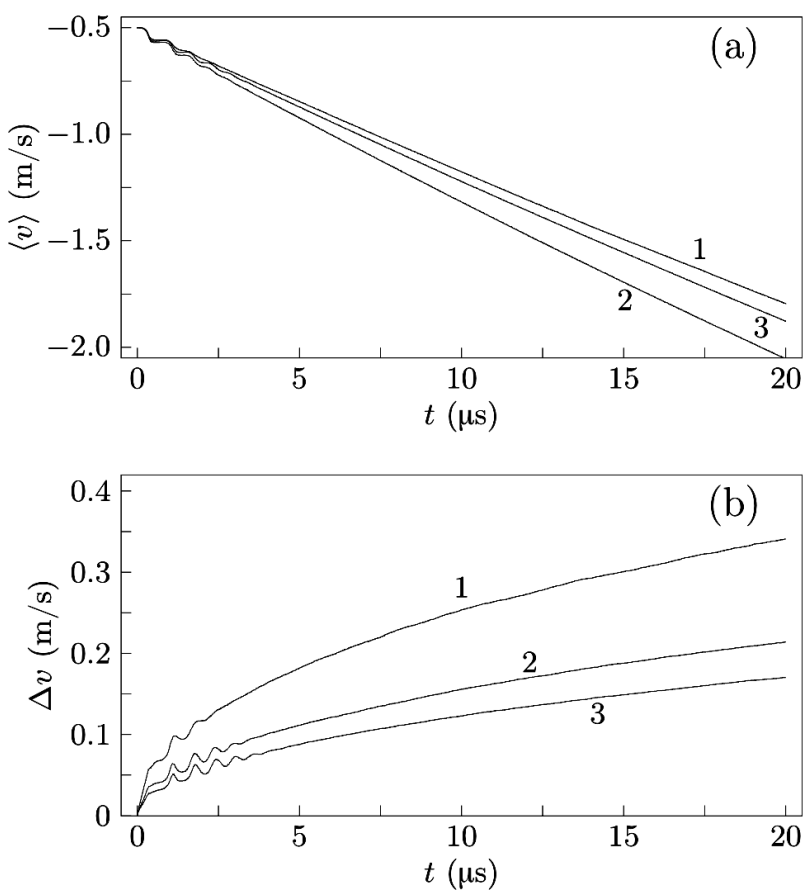

Fig. 2. Time dependences of the average velocity $(a)$ and the root-mean-square deviation of atomic velocities from the average value $(b)$. The Rabi frequency $\Omega_{0}$ of each of counterpropagating bichromatic waves is equal to $60 \mathrm{MHz}$, the initial velocity of atoms $v=-0.5 \mathrm{~m} / \mathrm{s}$, and the time delay between the waves at the point of initial atomic localization is $2.3 \mathrm{~ns}$. The parameter $G=2 \pi \times 10(1), 2 \pi \times 50(2)$, and $2 \pi \times 100 \mathrm{MHz}$ (3). Calculations were made for an ensemble of 10,000 atoms of ${ }^{133} \mathrm{Cs}$

and the momentum diffusion coefficient. Some examples of the calculated dependences of the light pressure force and the atomic velocity diffusion coefficient on the time delay between the counter-propagating stochastic waves are depicted in Fig. 3. It is evident that, in accordance with the results of work [5], if the Rabi frequency $\Omega_{0}$ and the inverse autocorrelation time $G$ of stochastic waves are fixed, there is a certain optimal value for the time shift $\tau$ between the counter-propagating waves, at which the force of light pressure on the atoms is maximum. By comparing the dependences shown in Fig. 3, one can see that the maximum force of light pressure at the fixed Rabi frequency $\Omega_{0}$ is achieved at a certain optimal value of the inverse correlation time, $G \sim \Omega_{0}$. In the case concerned (see Fig. 3, a), this is $G=30 \mathrm{MHz}$. At the same time, the diffusion coefficient $D_{v}$ decreases as the parameter $G$ grows (see Fig. $3, b$ ). Thus, by in- 

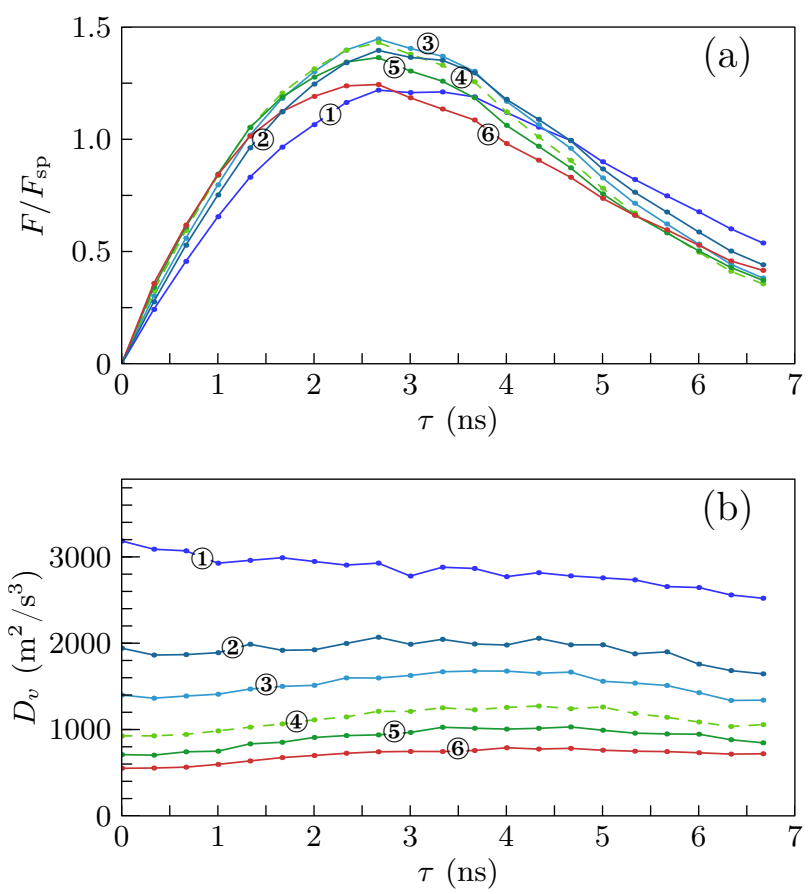

Fig. 3. Dependences of the light pressure force (in the $F_{s p}$ units) (a) and the coefficient of atomic velocity diffusion $D_{v}(b)$ on the time shift between the waves for various inverse times of correlation between the counter-propagating waves $G=2 \pi \times$ $\times 10(1), 2 \pi \times 20(2), 2 \pi \times 30$ (3) $, 2 \pi \times 50(4), 2 \pi \times 70$ (5), and $2 \pi \times 100 \mathrm{MHz}(6)$. The Rabi frequency $\Omega_{0}$ of each of the counter-propagating bichromatic waves equals $2 \pi \times 60 \mathrm{MHz}$, and the initial velocity of atoms is $-0.5 \mathrm{~m} / \mathrm{s}$. The force is directed toward the coordinate origin $(z=0)$. Calculations were made for an ensemble of 10,000 atoms of ${ }^{133} \mathrm{Cs}$

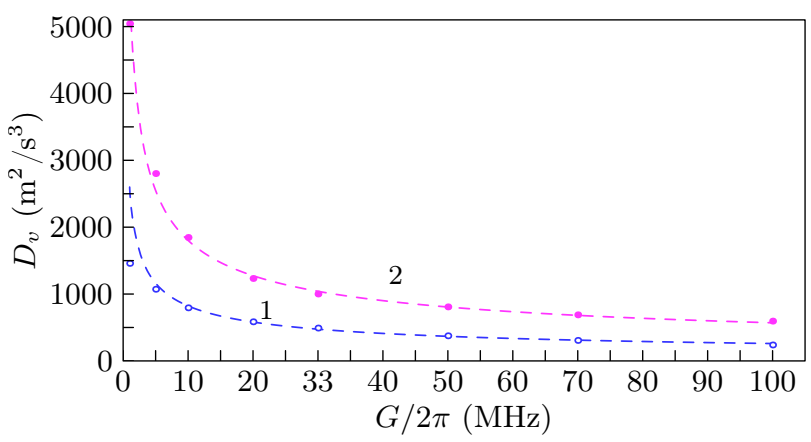

Fig. 4. Dependences of the atomic velocity diffusion coefficient $D_{v}$ on the inverse correlation time of counter-propagating waves $G$ (in megahertz units) for the Rabi frequency $\Omega_{0}=$ $=2 \pi \times 30(1)$ and $2 \pi \times 60 \mathrm{MHz}$ (2). The initial velocity of atoms is $-0.5 \mathrm{~m} / \mathrm{s}$. Calculations were made for an ensemble of 10,000 atoms of ${ }^{133} \mathrm{Cs}$. The time shift between the waves $\tau=12 \mathrm{~ns}$. The dashed curves correspond to $D_{v}=2.6 \times 10^{6} \sqrt{\frac{2 \pi}{G}}(1)$ and $5.7 \times 10^{6} \sqrt{\frac{2 \pi}{G}}$ (2)
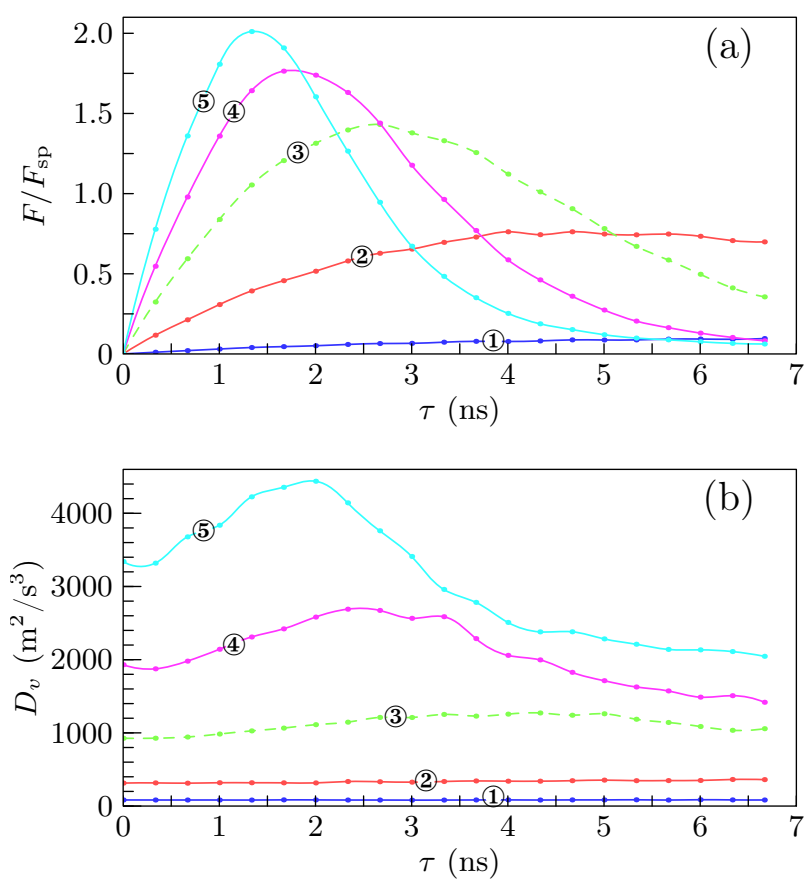

Fig. 5. Dependences of the light pressure force (in the $F_{s p}$ units) $(a)$ and the coefficient of atomic velocity diffusion $D_{v}$ (b) on the time shift between the waves for various Rabi frequencies $\Omega_{0}=2 \pi \times 10$ (1), $2 \pi \times 30$ (2), $2 \pi \times 60$ (3), $2 \pi \times 90$ (4), and $2 \pi \times 120 \mathrm{MHz}(5)$. The inverse correlation time of counterpropagating waves $G=2 \pi \times 50 \mathrm{MHz}$, and the initial velocity of atoms is $0.5 \mathrm{~m} / \mathrm{s}$. The force is directed toward the coordinate origin $(z=0)$. Calculations were made for an ensemble of 10,000 atoms of ${ }^{133} \mathrm{Cs}$

creasing $G$ at a fixed $\Omega_{0}$, we, at first, achieve a maximum value of the light pressure force accompanied by a simultaneous decrease of the parameter $D_{v}$. Then, as $G$ continues to grow, the intensity of light pressure at first decreases slowly (near the maximum) and, afterward, rather quickly. Simultaneously, the diffusion coefficient decreases monotonically and rather rapidly. In particular, for curves 2 to 5 , whose maximum amplitudes differ by no more than $10 \%$, the diffusion coefficient decreases by a factor of about two (see Fig. 3, b).

It is quite natural that the light pressure force has a maximum at a certain $G$ value. In work [5], it was shown that this maximum is associated with the delay between the counter-propagating waves at the point where the atom is located. As a result, if the parameter $G$ is small, the counter-propagating waves act almost in antiphase on the atom (practically, there is no delay), so that the force of light pressure on a sta- 
tionary or slowly moving atom is close to zero. In the case of large $G$, the counter-propagating waves fluctuate practically independently. Therefore, proceeding from the problem symmetry (the waves have the same average intensity), the force of light pressure should be small. At intermediate $G$ values, the correlation between the waves is substantial, and the intensity of the light pressure can be rather strong, exceeding Eq. (1) and reaching a maximum at a certain $G$ value.

The reduction of the diffusion coefficient $D_{v}$, as $G$ grows and $\Omega_{0}$ is fixed (see Fig. 3, b), qualitatively agrees with the behavior of $D_{v}$ at a low-intensity laser radiation [Eq. (32)]. At the same time, the origins of the diffusion coefficient reduction in the case $\Omega_{0} \ll \gamma$, when formula (32) is valid, and in the case $\Omega_{0} \gg \gamma$, which is illustrated in Fig. 3, are different. Indeed, under a low-intensity laser radiation, the momentum diffusion coefficient is proportional to the excited state population $n_{2}[6]$ :

$D=(\hbar k)^{2} \gamma n_{2}$.

By comparing Eqs. (32) and (33), one can see that, in the case of low-intensity counter-propagating waves, the decrease of the momentum diffusion coefficient occurs due to the reduction in the excited state population, as the fluctuation rate $G$ increases. In the case of high-intensity laser radiation, $\Omega_{0} \gg \gamma$ (the plots in Fig. 3), the diminishing of the diffusion coefficient can no longer be explained by the reduction in the excited state population. According to the results of our calculations corresponding to the curves shown in Fig. 3, the population $n_{2}$ changes insignificantly for various $G$ values, being close to $n_{2} \approx 0.45$. If Eq. (33) with $\Omega_{0}=2 \pi \times 60 \mathrm{MHz}$ had been valid for the momentum diffusion coefficient $D$, then $D_{v}$ would have been close to $170 \mathrm{~m}^{2} / \mathrm{s}^{3}$ for all curves in Fig. 3, b. Actually, the dependences exhibited in this figure correspond to much larger values of $D_{v}$. Such $D_{v}$ values may probably be associated with the processes of stimulated radiation emission and absorption, when the atom interacts with the fluctuating fields of counterpropagating stochastic waves. As a result, the motion of the atom in the momentum space looks like the Brownian one, but with the step decreasing with the increasing $G$, which can explain the reduction of the momentum diffusion coefficient.

Of course, our speculations about an analogy with the Brownian motion are not sufficient to establish a relationship between $D_{v}$ and $G$. To elucidate this issue, we numerically simulated the dependence $D_{v}(G)$ for two values of the Rabi frequency: $\Omega_{0}=2 \pi \times 30$ and $2 \pi \times 60 \mathrm{MHz}$. To "switch-off" the effects of mutual correlation between the counter-propagating waves, the calculations were made for a rather large time delay between the waves, $\tau=2 z / c=12 \mathrm{~ns}(z=1.8 \mathrm{~m})$. The results obtained are shown in Fig. 4 . One can see that the obtained dependences are described well by the expression $D_{v}=A / \sqrt{G}$ if $G>\gamma$. Since $1 / G$ is the wave autocorrelation time, we may assert that the coefficient of momentum diffusion is proportional to the square root of this parameter at $\Omega_{0} \gg \gamma$. At a lower intensity of the laser radiation $\left(\Omega_{0}=2 \pi \times 30 \mathrm{MHz}\right)$, a deviation from the given approximation formula is observed, in accordance with the fact that, at low intensities, the form of the dependence $D_{v}(G)$ has to change from $D_{v} \propto G^{-\frac{1}{2}}$ to $D_{v} \propto G^{-1}$.

Finally, as an appendix to Fig. 3, let us compare the dependences of the momentum diffusion coefficient on the delay time between the waves obtained for various intensities of counter-propagating waves, but for the same $G$ (see Fig. 5). The dashed curves in Figs. 3 and 5 correspond to the same parameter set $\left(\Omega_{0}=2 \pi \times 60 \mathrm{MHz}, G=2 \pi \times 50 \mathrm{MHz}\right)$. In Fig. 5, unlike Fig. 3 and in accordance with the results of work [5], there is no optimal value of the ratio $\Omega_{0} / G$, at which the force acting on an atom would be maximum. Instead, the force maximum increases monotonically together with the Rabi frequency. The force maximum and the maximum of the diffusion coefficient shift toward smaller $\tau$ values with the growth of $\Omega_{0}$. From Fig. 5, one can see that the diffusion coefficient increases, as the Rabi frequency increases and the $G$ value is fixed. At the same time, it decreases as $G$ increases and $\Omega_{0}$ is fixed (see Fig. 3).

\section{Conclusions}

Using cesium atoms as an example, the dependence of the momentum diffusion coefficient for atoms in the fields of two counter-propagating stochastic light waves on the delay between those waves has been studied and compared with analogous dependences obtained for the light pressure force. The results demonstrate that both the light pressure force and the momentum diffusion coefficient have a maximum at a certain delay value, with the maximum of the momentum diffusion coefficient being less pro- 
nounced. If the laser radiation intensity is constant, there is an optimal value for the inverse autocorrelation time $G$ of counter-propagating waves, at which the force is maximum. At the same time, the momentum diffusion coefficient monotonically decreases, as $G$ increases and the laser radiation intensity is constant. We have found that if the time delay between the waves is rather large, so that the amplitude fluctuations in the waves can be considered as independent, and the intensity of the laser radiation is large $\left(\Omega_{0} \gg \gamma\right)$, the momentum diffusion coefficient is proportional to $G^{-\frac{1}{2}}$. In other words, under those conditions, the momentum diffusion coefficient becomes proportional to the square root of the field auto-correlation time. This dependence substantially differs from the dependence $D_{v} \propto G^{-1}$ obtained by us earlier for the case of low-intensity fields [6].

We also compared the dependence of the momentum diffusion coefficient on the wave delay time at various intensities of counter-propagating waves, but at the same $G$ value. It is found that the diffusion coefficient increases together with the Rabi frequency. At the same time, it decreases, if $G$ increases and $\Omega_{0}$ is fixed.

This work was supported in the framework of the target program of scientific researches of the Division of physics and astronomy of the National Academy of Sciences of Ukraine "Study of fundamental physical and astronomical processes, and prospects of their practical application" (project No. 1.4.VTs/188).

1. V.S. Voitsekhovich, M.V. Danileiko, A.M. Negriyko, V.I. Romanenko, L.P. Yatsenko. Light pressure on atoms in the field of the counter-propagating amplitude-modulated waves. Ukr. Fiz. Zh. 36, 1042 (1991) (in Russian).

2. V.S. Voitsekhovich, M.V. Danileiko, A.M. Negriyko, V.I. Romanenko, L.P. Yatsenko. Light pressure on atoms in the field of the resonance to the atomic transition counterpropagating amplitude- and frequency-modulated waves. Ukr. Fiz. Zh. 18, 1100 (1991) (in Russian).

3. E.R. Orap, V.I. Romanenko, L.P. Yatsenko. Light pressure on the atoms in the field of counterpropagating light waves with sinusoidal and stochastic phase modulation. Ukr. J. Phys. 48, 211 (2003).

4. V.I. Romanenko. Light pressure on atoms in the field of counterpropagating waves with stochastic amplitudes. $U k r$. J. Phys. 50, 438 (2005).

5. V.I. Romanenko, B.W. Shore, L.P. Yatsenko. Forces exerted on atoms by stochastic laser fields. Opt. Commun. 268, 121 (2006).
6. V.I. Romanenko, L.P. Yatsenko. Trapping of atoms by the counter-propagating stochastic light waves. Opt. Commun. 392, 239 (2017).

7. V.S. Voitsekhovich, M.V. Danileiko, A.M. Negriyko, V.I. Romanenko, L.P. Yatsenko. Light pressure on atoms in counterpropagating amplitude-modulated waves. Sov. Phys. Tech. Phys. 33, 690 (1988).

8. J. Söding, R. Grimm, Y.B. Ovchinnikov, P. Bouyer, C. Salomon. Short-distance atomic beam deceleration with a stimulated light force. Phys. Rev. Lett. 78, 1420 (1997).

9. L. Yatsenko, H. Metcalf. Dressed-atom description of the bichromatic force. Phys. Rev. A 70, 063402 (2004).

10. V.G. Minogin, V.S. Letokhov, Laser Light Pressure on Atoms (Gordon and Breach, 1987).

11. K. Mølmer, Y. Castin, J. Dalibard. Monte Carlo wavefunction method in quantum optics. J. Opt. Soc. Am. B 10, 524 (1993).

12. A.T. Georges, P. Lambropoulos. Saturation and Stark splitting of an atomic transition in a stochastic field. Phys. Rev. A 20, 991 (1979).

13. A.T. Georges. Resonance fluorescence in Markovian stochastic fields. Phys. Rev. A 21, 2034 (1980).

14. W. Horsthemke, R. Lefever. Noise-Induced Transitions: Theory and Application in Physics, Chemistry, and Biology (Springer, 1984).

15. H.J. Metcalf, P. van der Stratten. Laser Cooling and Trapping (Springer, 1999).

16. B.W. Shore. The Theory of Coherent Atomic Excitation, Vol. 1 (Wiley, 1990).

17. R.F. Fox, I.R. Gatland, R. Roy, G. Vemuri. Fast, accurate algorithm for numerical simulation of exponentially correlated colored noise. Phys. Rev. A 38, 5938 (1988).

18. G. Vemuri, R. Roy. Effect of injected field statistics on transient dynamics of an injection seeded laser. Opt. Commun. $\mathbf{7 7}, 318$ (1990).

Received 30.05.18

Translated from Ukrainian by O.I. Voitenko

В.І. Романенко, О.Г. Удовицъка,

В.М. Ходаковсъкий, Л.П. Яценко

ІМПУЛЬСНА ДИФУЗІЯ АТОМІВ У ПОЛІ

ЗУСТРІЧНИХ СТОХАСТИЧНИХ СВІТЛОВИХ ХВИЛЬ

$\mathrm{P}$ е $з$ ю м е

Досліджено імпульсну дифузію атомів у полі зустрічних світлових стохастичних хвиль, одна з яких повторює іншу з деякою затримкою. Показано, що параметрам взаємодії атома з полем, оптимальним для досягнення великого світлового тиску в таких полях, відповідає зростання коефіцієнта імпульсної дифузії. У полі інтенсивних хвиль, що описуються моделлю стохастичного поля, коефіцієнт імпульсної дифузї пропорційний квадратному кореню з часу автокореляції хвиль. Внутрішній стан атомів моделювався методом Монте-Карло для хвильової функції. Чисельне моделювання проведено для атомів цезію. 